\title{
Colorectal liver metastases: surgery versus thermal ablation (COLLISION) - a phase III single-blind prospective randomized controlled trial
}

Robbert S. Puijk ${ }^{1 *}$, Alette H. Ruarus', Laurien G. P. H. Vroomen', Aukje A. J. M. van Tilborg ${ }^{1}$, Hester J. Scheffer', Karin Nielsen², Marcus C. de Jong', Jan J. J. de Vries', Babs M. Zonderhuis², Hasan H. Eker', Geert Kazemier², Henk Verheul ${ }^{3}$, Bram B. van der Meijs ${ }^{1}$, Laura van Dam ${ }^{1}$, Natasha Sorgedrager ${ }^{1}$, Veerle M. H. Coupé

Petrousjka M. P. van den Tol' ${ }^{2}$ Martijn R. Meijerink ${ }^{1}$ and COLLISION Trial Group

\begin{abstract}
Background: Radiofrequency ablation (RFA) and microwave ablation (MWA) are widely accepted techniques to eliminate small unresectable colorectal liver metastases (CRLM). Although previous studies labelled thermal ablation inferior to surgical resection, the apparent selection bias when comparing patients with unresectable disease to surgical candidates, the superior safety profile, and the competitive overall survival results for the more recent reports mandate the setup of a randomized controlled trial. The objective of the COLLISION trial is to prove noninferiority of thermal ablation compared to hepatic resection in patients with at least one resectable and ablatable CRLM and no extrahepatic disease.
\end{abstract}

Methods: In this two-arm, single-blind multi-center phase-III clinical trial, six hundred and eighteen patients with at least one CRLM $(\leq 3 \mathrm{~cm})$ will be included to undergo either surgical resection or thermal ablation of appointed target lesion(s) $(\leq 3 \mathrm{~cm})$. Primary endpoint is OS (overall survival, intention-to-treat analysis). Main secondary endpoints are overall disease-free survival (DFS), time to progression (TTP), time to local progression (TTLP), primary and assisted technique efficacy (PTE, ATE), procedural morbidity and mortality, length of hospital stay, assessment of pain and quality of life (QoL), cost-effectiveness ratio (ICER) and quality-adjusted life years (QALY).

Discussion: If thermal ablation proves to be non-inferior in treating lesions $\leq 3 \mathrm{~cm}$, a switch in treatment-method may lead to a reduction of the post-procedural morbidity and mortality, length of hospital stay and incremental costs without compromising oncological outcome for patients with CRLM.

Trial registration: NCT03088150, January 11th 2017.

Keywords: Colorectal cancer, Colorectal liver metastases (CRLM), Liver metastases, Hepatic resection, Liver surgery, Thermal ablation, Radiofrequency ablation (RFA), Microwave ablation (MWA)

\footnotetext{
* Correspondence: r.puijk@vumc.nl

${ }^{1}$ Department of Radiology and Nuclear Medicine, Amsterdam University

Medical Centres (location VUmc), de Boelelaan 1117, 1081, HV, Amsterdam,

The Netherlands

Full list of author information is available at the end of the article
}

(c) The Author(s). 2018 Open Access This article is distributed under the terms of the Creative Commons Attribution 4.0 International License (http://creativecommons.org/licenses/by/4.0/), which permits unrestricted use, distribution, and reproduction in any medium, provided you give appropriate credit to the original author(s) and the source, provide a link to the Creative Commons license, and indicate if changes were made. The Creative Commons Public Domain Dedication waiver (http://creativecommons.org/publicdomain/zero/1.0/) applies to the data made available in this article, unless otherwise stated. 


\section{Background}

Colorectal cancer is the third most common malignancy worldwide and the second most common cause of cancer related death in developed countries $[1,2]$. Approximately half of the patients will develop colorectal liver metastases (CRLM). Only 10-15\% are considered eligible for partial hepatectomy $(\mathrm{PH})$, due to (1) an impaired general health status, (2) a history of extensive abdominal surgery, (3) the presence of lesions with an unfavourable anatomical location or (4) an insufficient future liver remnant to resect all lesions [3-7]. These patients are usually treated with chemotherapy and/or thermal ablation, alone or in combination with $\mathrm{PH}$.

Contradictory to most cancer types, long-term survival and even cure is possible in a subset of patients with CRLM [8]. Median overall survival (OS) of untreated CRLM (receiving only symptomatic therapy) is 4.5-12 months [9]. Chemotherapy has improved OS, but OS remains humble at 15-20 months $[10,11]$.

Surgical resection of the metastases has long been considered the only curative treatment option. In the past few years, radiofrequency ablation (RFA) and microwave ablation (MWA) techniques have rapidly worked their way into clinical guidelines for treatment of unresectable liver tumours [12]. For solitary small (< $2 \mathrm{~cm}$ ) hepatocellular carcinomas, international guidelines have shifted from surgery to minimally-invasive percutaneous thermal ablation because local control rates have reached $100 \%$ [6, 13-17].

Four recent series reported a comparable OS for thermal ablation versus surgical resection [14, 18-20]. These results have led to the discussion whether or not thermal ablation - being less invasive - should be favoured over resection for smaller lesions. Despite this, 5-year OS $(25-55 \%)$ of thermal ablation for patients with unresectable CRLM has been labelled inferior to surgical resection for patients with resectable CRLM according to previous meta-analyses and systematic reviews [21-29]. These results should be interpreted with caution due to the apparent selection bias. At this point, there are no high-quality randomized controlled trials comparing thermal ablation to surgical resection for resectable CRLM, even though the need has previously been suggested by various authors [8, 30, 31]. To prove non-inferiority, we have designed a two-arm single-blind multi-center phase-III randomized controlled trial comparing surgical resection (standard of care) to thermal ablation (experimental arm) for resectable and ablatable CRLM $\leq 3 \mathrm{~cm}$.

\section{Design/methods}

Design

COLLISION is a national, single-blind, multi-center, phaseIII trial that is organized by the Amsterdam University
Medical Centres (location VUmc) in Amsterdam, the Netherlands. The study is accommodated by the Dutch Colorectal Cancer Group (DCCG) and formally endorsed by the Dutch national covering patient federations, Dutch national societies for interventional radiology (NVIR), radiology $(\mathrm{NVvR})$, surgery $(\mathrm{NVvH})$, and the liver surgery working group (WLC).). Patients will be recruited in, at least sixteen, high-volume centres for liver surgery throughout the Netherlands: Amsterdam UMC (location VUmc), Amsterdam; Amsterdam UMC (location AMC), Amsterdam; Leiden University Medical Center (LUMC), Leiden; Radboud University Medical Center, Nijmegen; Maastricht University Medical Center (MUMC), Maastricht; Antoni van Leeuwenhoek (AvL), Amsterdam; Medical Center Leeuwarden (MCL), Leeuwarden; Ziekenhuis Gelderse Vallei (ZGV), Ede; Isala Klinieken, Zwolle; Deventer Ziekenhuis, Deventer; Westfriesgasthuis, Hoorn; Erasmus Medical Center (EMC), Rotterdam; Jeroen Bosch Ziekenhuis (JBZ), Den Bosch; Medisch Spectrum Twente (MST), Enschede; Onze Lieve Vrouwe Gasthuis (OLVG), Amsterdam; University Medical Center (UMCU), Utrecht). The protocol has been approved by the Medical Ethical Review Board (METc) of the Amsterdam University Medical Centres (location VUmc) for Dutch national approval (no. 2016.561). The trial is investigator-sponsored by Medtronic PLC, independent of industry and registered at clinicaltrials.gov (NCT03088150, January 11th 2017). The trial will be conducted in accordance with the Declaration of Helsinki (64th version, October 2013) and the guidelines for Good Clinical Practice (GCP). The in- and exclusion criteria are summarized in Table 1.

The total duration of the study is around 13 years considering an inclusion time of 3 years and a minimum follow-up period of 10 years. All participants will provide written informed consent.The flow diagram of is shown in Fig. 1.

\section{Start COLLISION trial}

Inclusion, randomization and treatments started in two hospitals by the end of 2017. Due to formal approval procedures by local authorities, only AmsterdamUMC (location VUmc) and ZGV Ede were able to include patients from the beginning. From May 2018, RadboudUMC, LUMC, MCL, Isala Klinieken and Westfries Gasthuis were also able to participate. Numerous other Dutch high-volume liver centres, which are mentioned above, are waiting for local approval and will participate in the near future.

\section{Eligibility}

Potential candidates will be registered and undergo routine pre-procedural work-up: baseline full blood examination, carcinoembryonic antigen (CEA), bone marrow, liver, and 
Table $1 \mathrm{ln}$ - and exclusion criteria

\begin{tabular}{ll}
\hline Inclusion criteria & Exclusion criteria \\
\hline Histological documentation of & No target lesions suitable for both \\
primary colorectal tumour & resection and ablation \\
Age $>18$ years & Radical treatment unfeasible or \\
& unsafe (e.g. insufficient future liver \\
& remnant [FLR])
\end{tabular}

At least one CRLM size $\leq 3 \mathrm{~cm}$ eligible for both surgical resection and thermal ablation (target lesions)

Additional unresectable CRLM should be $\leq 3 \mathrm{~cm}$ and ablatable

Additional unablatable CRLM should be resectable

Maximum number of CRLM 10

Resection for resectable lesions considered possible obtaining negative resection margins (RO) and preserving adequate liver reserve

Resectability and ablatability should be re-confirmed by intra-operative ultrasound (IOUS) and full surgical exploration

Eastern Cooperative Oncology Group status (ECOG) 0-2

American Society of

Anesthesiologists (ASA) grade 1-3

Life expectancy of at least

12 weeks

Adequate bone marrow, liver, and renal function as assessed by local usual laboratory tests. As usual,

these results should be judged by

the local investigator and should be

conducted within 7 days prior to

definite inclusion.

Written informed consent

renal function -, anaesthetic review, ceCT of the chest and abdomen and either an upper abdominal ceMRI or a total body ${ }^{18}$ F-FDG PET-CT using upper abdominal ceMRI as problem solver. Patients with $\geq 1$ resectable and ablatable CRLM ( $\leq 3 \mathrm{~cm})$, no extrahepatic disease and a good performance status (WHO 0-2) are considered eligible. Supplementary resections for resectable lesions $>3 \mathrm{~cm}$ and thermal ablations for unresectable CRLM $\leq 3 \mathrm{~cm}$ are allowed with a maximum number of CRLM of 10 (Table 1 ).

Eligible patients will be stratified into low-, intermediate- and high disease burden after assessment by an expert panel (Fig. 1). The panel, consisting of at least two diagnostic radiologists, two interventional radiologists and two hepatobiliary and/or oncological surgeons, will appoint lesions that are resectable and ablatable as target lesions, resectable and unablatable lesions as unablatable lesions and ablatable but unresectable lesions as unresectable lesions. All unablatable lesions should be resectable and all unresectable lesions should be $\leq 3 \mathrm{~cm}$ and ablatable. Because definitions of resectability and ablatability can vary dramatically from one center to the other and from one specialist to the other, the panel has to agree with the treating physicians' treatment plan. If the panel disagrees, the panel and the treating physicians must reach consensus before the patient can be enrolled.

\section{Methods}

Participating centres should have extensive experience in the field of both hepatic surgery and thermal liver tumour ablation, defined as performing $\geq 20$ procedures annually. Treating surgeons and interventional radiologists should be board certified and have performed and/or supervised $\geq 100$ procedures.

\section{Inclusion}

After having obtained written informed consent by the outpatient clinic doctor, patients will be formally included. The patient should be scheduled to undergo the procedure within a time-frame of maximum 6 weeks hereafter. Patients suitable for either laparoscopic resection or percutaneous ablation (Subgroup A, low disease burden; 1-3 target lesions) will be randomized prior to the procedure. All other patients will undergo open laparotomy with surgical inspection of the abdominal cavity and intra-operative ultrasound (IOUS).

\section{Exclusion (drop-outs)}

Despite improvements in preoperative imaging technology, the intraoperative use of ultrasonography remains of crucial importance [32]. The detection rate of preoperatively unknown lesions is still high (up to 50\%) with considerable consequences on treatment strategy [32]. Following surgical inspection and IOUS the inclusion criteria need to be reconfirmed prior to randomization. If (1) a radical procedure is no longer considered safe or feasible, if (2) > 10 CRLM are present, if (3) extrahepatic disease is detected, or if (4) no lesion can be appointed as target lesion, the patient cannot be included in the study and will be treated as non-study object. Additional CRLM suitable for both resection and ablation $\leq 3 \mathrm{~cm}$ will be appointed as new target lesions. Additional unresectable lesions $\leq 3 \mathrm{~cm}$ that are suitable for thermal ablation should be ablated if possible and vice versa additionally detected unablatable lesions should be resected. Lesions, preprocedurally appointed as target lesions, that prove unsuitable for one treatment modality based on IOUS lose their status and should be treated with the alternate modality (lesion shifts from target lesion to unablatable or unresectable lesion). 


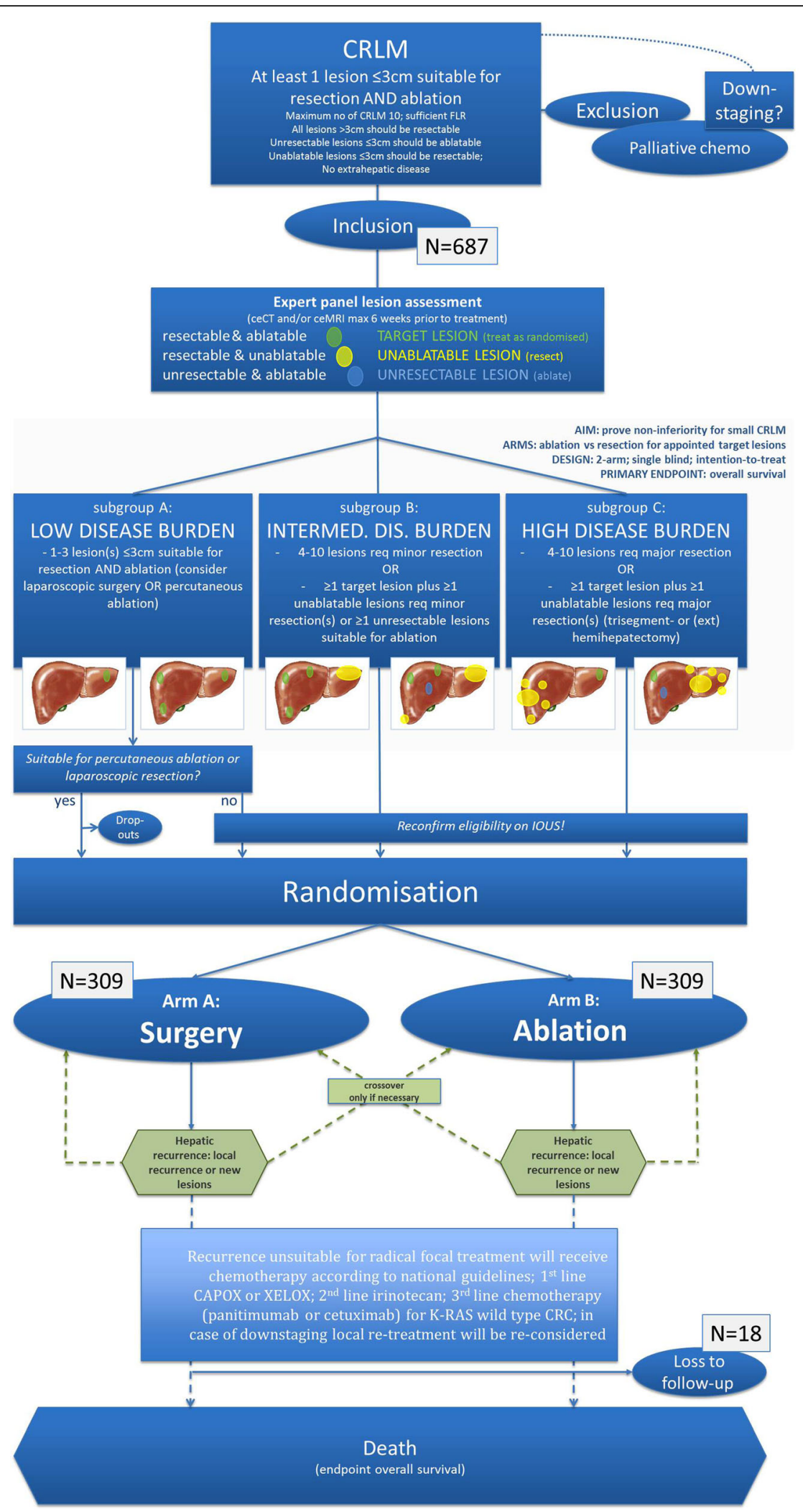

Fig. 1 Flow diagram of study procedure 


\section{Laparotomy}

The surgical explorative procedure of participants in this study is identical to standard procedures for non-study objects. A right subcostal incision is performed. The abdominal cavity will be explored in order to exclude extrahepatic tumour manifestations. An IOUS to exclude additional CRLM and for final confirmation of resectability will always be performed.

\section{Randomization}

Patients with limited disease burden (max. 3 lesions $\leq 3 \mathrm{~cm}$ ) that are suitable for percutaneous ablation or laparoscopic resection will be randomized, prior to the procedure, into one of two arms, arm A and arm B. All other patients will undergo laparotomy with IOUS and surgical inspection and will, if still considered eligible, be randomized during general anaesthesia. Patients included in study arm A will undergo resection of hepatic metastases, allowing thermal ablation for additional unresectable lesions. Patients included in study arm B will undergo ultrasound guided thermal ablation of hepatic metastases, allowing resection for additional unablatable lesions (Fig. 1).

Randomization is centralized and performed through a web-based module (Castor EDC ${ }^{\oplus}$ ) [33], which is accessible 7 days a week, $24 \mathrm{~h}$ per day]. For open procedures randomization will be performed shortly after surgical inspection and IOUS with the patient under general anaesthesia. Both the experimenter(s) and the participant will be unaware of the eventual treatment arm prior to the procedure; after the procedure the patient will remain unaware (single-blind).Because follow-up imaging will reveal the nature of the focal therapy and because knowledge about the actual procedure and pathological confirmation of tumour free margins is required to reliably assess ${ }^{18}$ F-FDG PET-CT follow-up scans, the panel's diagnostic abdominal radiologists and nuclear physicians need to be informed about the specific treatment history.

Changes in insights detected after randomization do not allow patient's exclusion. These patients will remain in their originally appointed group according to the intention-to-treat analysis. For example, if, after being randomized into the resection arm, a target lesion proves unresectable during surgical tissue preparation and dissection, the patient will remain in arm A (resection) even if the lesion was eventually ablated or left untreated.

\section{Surgical resection}

In case of randomization to surgical resection, the surgeon will remove all target lesions as well as all additional unablatable lesions. The extent of the resection, the resection margins and the specific technique is at the discretion of the performing liver surgeon. Complications encountered during the procedure will be noted.
Postoperative care will be on the recovery and subsequently on either the surgery ward or medium care whenever deemed necessary. General 'resectability' criteria are shown in Table 2.

\section{Thermal ablation}

The safety, feasibility and preferred type of thermal ablation(s) is at the discretion of the interventional radiologist. Ablations are performed according to the CIRSE quality improvement guidelines with an intentional tumour free ablation margin of at least $1 \mathrm{~cm}$ [34].

Patients with limited disease burden (max. 3 lesions $\leq 3 \mathrm{~cm}$ ) and no contra-indications for a percutaneous approach will be randomized prior to the procedure. Contra-indications for a percutaneous approach are proximity of critical structures. To avoid collateral damage to intestines a minimum distance to the stomach, small bowel and colon of $15 \mathrm{~mm}$ should be respected. Laparoscopic approach is allowed. Pneumo- and hydrodissections are allowed. Pringle-manoeuvres are not allowed.

Following percutaneous ablations, a ceCT or ceMRI should always be performed for ablated lesions $>2 \mathrm{~cm}$ and for lesions $0-2 \mathrm{~cm}$ with radiologically unclear margins after the ablation. Unequivocal local site residues or insufficient tumour-free margins should be re-ablated (completion ablation) within 4 weeks after the initial ablation. If re-ablated within 4 weeks, the residue/insufficient margins count as

Table 2 General 'resectability' and 'ablatability' criteria

\begin{tabular}{ll}
\hline General 'resectability criteria' & General 'ablatability criteria' \\
\hline No size limit & Maximum CRLM size $\leq 3 \mathrm{~cm}$ \\
Aiming at negative (R0) margins & $\begin{array}{l}\text { Aiming at a tumour free margin } \\
\text { of }>10 \mathrm{~mm}\end{array}$ \\
$\begin{array}{ll}\text { Leave sufficient FLR (> 20\% normal } \\
\text { functioning liver parenchyma; }\end{array}$ & $\begin{array}{l}\text { Leave sufficient FLR ( }>20 \% \text { normal } \\
>30 \% \text { post-chemotherapy) }\end{array}$ \\
$\begin{array}{ll}\text { Portal vein embolization of the } \\
\text { (most) affected liver lobe marenchyma; }\end{array}$ \\
$\begin{array}{l}\text { considered for patients with } \\
\text { insufficient FLR }\end{array}$ & $\begin{array}{l}\text { To preserve the major bile ducts } \\
\text { duct) a minimum distance (lesion }\end{array}$ \\
& to major bile duct) of 15 mm is \\
& required
\end{tabular}

At least one of three hepatic veins should be preserved and both the portal venous and hepatic arterial blood flow in the future liver remnant should be remain unharmed

Approachable surgical field, without extensive scar formation, major surgical adhesions and/or intestinal herniations (risk of major morbidity estimated $>20 \%$; risk of mortality estimated $>5 \%$ )

Radical ablation(s) with or without surgical resections for additional unablatable lesions

To avoid collateral damage to the intestines a minimum distance to the stomach, small bowel and colon of $15 \mathrm{~mm}$ should be pursued in open procedures and respected in percutaneous procedures; Pneumo- or hydrodissections to shift bowels are allowed

Maximum total number of Maximum total number of CRLM 10 
technically unsuccessful ablations, but not as a tumour recurring event when assessing the primary technique efficacy, local progression-free and disease-free survival. Patients with limited disease burden plus a contra-indication for both percutaneous ablation and for laparoscopic surgery and patients with intermediate or high disease burden will be randomized during open laparotomy.

The probes are connected to compatible and commercially available generators. Ablations will be performed according to the protocols provided by the manufacturers. If necessary, the needle electrodes will be repositioned for one or more overlapping ablations. The proximity of a large portal or systemic vein or hepatic artery is no contraindication for performing the thermal ablation.

The definition of a technically successful ablation is based upon the specific protocols established by the device manufacturers in combination with an immediate post-procedurally performed US (fully hyperechoic ablation zone with an intentional margin of at least $1 \mathrm{~cm}$ ) [7]. Necessity for re-ablations and/or needle repositioning will be judged by the performing interventional radiologist. Postoperative care will be on the recovery room and subsequently on either the surgery ward or medium care whenever deemed necessary. A quality-control ceCT can be performed within 1-6 weeks after the initial treatment to assess for a completion-procedure [7]. General 'ablatability' criteria are shown in Table 2.

\section{Follow-up}

Conferring to national guidelines follow-up will include imaging, laboratory tests including tumour markers (CEA) and clinical examination every 3 months for the first year and every 6 months hereafter. Follow-up cross-sectional imaging should include at least an abdominal ceCT or upper abdominal ceMRI at the given time-points. Participating centres are free to add ${ }^{18} \mathrm{~F}$-FDG PET-CTs at specific time-points or to use alternating specific modalities, as long as the follow-up protocol is pre-approved by the trial coordinators and as long as follow-up imaging is identical for both treatment arms. Quality of life questionnaires will be assessed at baseline, every 3 months for the first year and every 6 months hereafter accordingly. Data will be collected in Castor EDC ${ }^{\bullet}$ [33], only available for related research investigators.

\section{Primary and secondary objectives}

The main objective is to prove non-inferiority of thermal ablation compared to hepatic resection in patients with at least one resectable and ablatable CRLM $(\leq 3 \mathrm{~cm})$ and no extrahepatic disease. Primary endpoint is OS. Main secondary endpoints are overall disease-free survival (DFS), time-to-progression (TTP), time-to-local-progression (TTLP), primary and assisted technique efficacy (PTE, ATE), procedural morbidity and mortality, length of hospital stay, assessment of pain and quality of life (QoL), cost-effectiveness ratio (ICER) and quality-adjusted life years (QALY).

Pain analysis will be performed using visual analogue scale questionnaires (VAS) assessed prior to, directly after and every 3 months after local treatment; administered pain medication will be registered. Quality of life analysis will be performed using European Organisation for Research and Treatment of Cancer Quality of Life questionnaires (EORTC-QLQ-CR29, EORCT QLQ-C30, EQ-5D) prior to, and every 3 months after local treatment. Patients who complete the quality-of life questionnaires at baseline and at least once during treatment and follow-up will be included in the analysis. The largest decrease in quality of life with respect to baseline will be calculated. The Wilcoxon rank sum test will be used to detect statistical differences between the two treatment arms.

\section{Sample size calculation and statistical considerations}

We hypothesize (null-hypothesis) that thermal ablation is non-inferior to surgical resection for the selected patient groups in terms of the primary objective (OS). The Cox proportional hazards model (1-sided; non-inferiority or superiority) is used for sample size calculations (Table 3). Given the superior safety profile we consider a hazard ratio (HR) of 1.3 to represent the upper limit of non-inferiority (non-inferiority margin). An HR of 1.3 corresponds to a $56.5 \%$ chance of the ablated patients to die first $((\mathrm{P}=\mathrm{HR} /$ $(1+\mathrm{HR})=1.3 /(1+1.3)=0.565 \quad(56.5 \%))$. We will have reached $60 \%$ of events (death) approximately 6.5 years after having included the last patient (overall probability of event, $\mathrm{pE}=0.6$ ). The calculated sample size therefore is 599 (NS). To account for a $10 \%$ drop-out ratio $(\mathrm{NDO}=69)$ prior to randomization and a $3 \%$ loss to follow-up $(\mathrm{NLTFU}=18)$ after randomization we need to recruit 687 patients (NI). A total number of 618 patients (687-69 (NDO)) will be randomized (NR) into one of two arms: arm A will undergo surgical resection $(n=309)$ and

Table 3 Sample size calculation

\begin{tabular}{ll}
\hline Significance level $(a)$ & 0.05 \\
\hline Power (1- $\beta$ ) & 0.80 \\
Hazard Ratio (HR), $\theta$ (non-inferiority margin) & 1.3 \\
Null-Hypothesis Hazard Ratio, $\theta_{0}$ & 1.0 \\
Recruitment time/study accrual (months) & 36 \\
Follow-up time (months) & 60 \\
Ratio control vs. experimental: $\mathrm{m}_{2} / \mathrm{m}_{1}$ & 1.0 \\
Total sample size $\left(\mathrm{N}_{\mathrm{S}}\right) /$ total number to be randomized $\left(\mathrm{N}_{\mathrm{R}}\right)$ & 599 \\
Accounting for 3\% loss to follow-up after randomization $\left(\mathrm{N}_{\mathrm{LTFU}}\right)$ & 18 \\
Accounting for $10 \%$ drop-out ratio pre-randomization $\left(\mathrm{N}_{\mathrm{DO}}\right)$ & 69 \\
Initial pre-randomization sample size - number of included & 687 \\
patients $\left(\mathrm{N}_{1}\right)$ & \\
\hline
\end{tabular}


arm B thermal ablation $(n=309)$ for appointed target lesions.

\section{Statistical methods}

All clinicopathological and procedural variables will be described and analysed. Continuous variables will be summarized with standard statistics including, means, standard deviations, medians and ranges. Categorical variables will be summarized with frequencies. When appropriate, box plots and cross tables will be used for descriptive statistics of continuous and categorical variables, respectively. $P$-values below 0.05 will be considered significant. All calculations will be generated by statistical package for social sciences software $\left(\mathrm{SPSS}^{\circ}\right)$. Calculation of the number of patients that will be needed to address our primary endpoint with a power of $80 \%$ and a $5 \%$ type I error rate is described in the sample size calculation section.

Univariate survival analysis will be performed using the Kaplan-Meier method. Differences in survival lengths will be analysed using the log rank test. To determine hazard ratios (HR) for multivariate analysis, Cox regression will be used. Significance of differences for continuous and categorical data will be analysed using the Mann-Whitney $\mathrm{U}$ test and Chi-square test respectively. When appropriate, box plots and cross tables will be used for descriptive statistics of continuous and categorical variables, respectively. OS will be estimated by the Kaplan-Meier method with corresponding two sided 95\% Cl's for survival proportions.

Primary and assisted technique efficacy rates (PTE, ATE) defined as the percentage of target lesions that have recurred after the initial local treatment and after additional local treatments regardless of the technique(s) used to treat the recurrence with a minimum follow-up period of 12 months after the last focal therapy;- Direct and indirect total cost of care for both treatment arms will be registered in the cost-effectiveness data collection matrix. Based on this matrix a cost-utility analysis, measured in terms of years of full health lived, using quality-adjusted life years will be prospectively calculated. Cost-effectiveness will be expressed as an ICER, the ratio of change in costs to the change in effects.

\section{Data monitoring}

The investigators believe that an independent data safety and monitoring committee (DSMB) is unnecessary given the much less invasive nature and superior safety profile of the experimental treatment arm (thermal ablation). An independent monitor committee (Clinical Research Bureau; $\mathrm{CRB}$ ) is appointed to safeguard the quality of all investigator-initiated studies. A quality officer from the CRB will monitor all study data according to Good Clinical Practice (GCP). The informed consent of selected individual participants will be checked. Source Data verification will be performed during onsite monitoring (to verify if all data on the Case Report Form are in accordance with the source data). The intensity of this verification is in relation to the risk associated with the intervention investigated, which is considered acceptable. For all subjects, the informed consent forms, the in- and exclusion criteria and the primary outcome (overall survival from the date of randomization to the date of death due to any cause) will be verified. The monitor will also verify if all (S)AE's are reported adequately and within the time that is determined by legal rules and regulations.

Shortly after beginning of the study the research group and epidemiologists will compose a detailed plan regarding futility and criteria to end the study prematurely. The interim analysis will be performed on the primary endpoint using a non-inferiority analysis. If at interim analysis, after having randomized $30 \%$ of the patients, the number of deaths due to treatment is significantly higher in patients included in the experimental arm B compared to patients included in the control arm A, the study will be ended prematurely. If the interim analysis shows a trend towards a type 1 or type 2 error, we will add a Data Safety and Monitoring Board (DSMB) to our study. A new interim analysis will be conducted after having randomized $50 \%$ of the patients.

\section{(Serious) adverse events ( $A E$ 's and $S A E$ 's) and serious adverse device effects (SADE)}

All serious adverse events that occur in the first 90-days after the procedure that are life threatening or result in death, both related and unrelated to the research, and serious adverse events that happen during complete study follow up, that are life threatening or result in death and are related (unlikely, possible, probable or definite) to the research according to one of the principal investigator, will be reported within 7 days after the responsible investigator has first knowledge of the adverse reaction. This is for a preliminary report with another 8 days for completion of the report. Relationship of the event to the research will be established by the primary investigator as: $1=$ Unrelated (clearly not related to the research), $2=$ Unlikely (doubtfully related to the research), $3=$ Possible (may be related to the research), 4 $=$ Probable (likely related to the research), $5=$ Definite (clearly related to the research). All participating clinicians will be made aware of the necessity to report (serious) adverse events to the principal investigators. The sponsor will report the SA(D)E's through the web portal ToetsingOnline.nl to the accredited EC that approved the protocol, within 15 days after the sponsor has first knowledge of the serious adverse events.

The expedited reporting will occur not later than 7 days after the responsible investigator has first knowledge of the 
adverse event. This is for a preliminary report with another 8 days for completion of the report.

The sponsor (also) has an insurance which is in accordance with the legal requirements in the Netherlands (Article $7 \mathrm{WMO}$ and the Measure regarding Compulsory Insurance for Clinical Research in Humans of July 1st, 2015). This insurance provides cover for damage to research subjects through injury or death related to study participation.

\section{Cost-effectiveness analysis}

General considerations For this clinical trial, a cost effectiveness (utility) analysis will be performed from a societal perspective, using a 3-year time horizon. The direct and indirect costs will be included. Direct costs taken into account will include treatment costs, cost of hospitalization, medication, imaging, laboratory testing and pathology.

Within the trial, resource use will be monitored and this will be linked to integral cost prices or Dutch tariffs.

Patient outcome analysis To assess indirect cost, patients will be asked to fill out the Productivity and Disease Questionnaire (PRODISC) every 6 months. To calculate total indirect costs, the friction cost approach will be used.

Cost analysis the primary health outcome measure in this economic evaluation will be the total quality adjusted life years (QALY) per trial arm. QALYS will be calculated by using the utility scores linked to the various health states of the EQ-5D; in essence the length of time a patient spends in a particular health condition is weighed by the corresponding utility. Missing data on costs and utilities will be imputed using multiple imputation. The difference in total costs and total QALYs in both arms will be used to calculate the incremental cost-effectiveness ratio (ICER): the cost per QALY gained (or cost-savings per QALY gained or lost), using the formula: ICER $=($ Cintervention - Ccontrol $) /($ QALYintervention - QALYcontrol). Cost and health effect will be discounted using the Dutch discount rates of $1.5 \%$ for health effects and $4 \%$ for costs. In addition, to allow comparison with international studies, discount rates of $3 \%$ for both health effects and costs will be used as well. To assess the impact of uncertainty, a probabilistic sensitivity analysis will be performed using the non-parametric bootstrap with 5000 replications. The results will be presented on cost-effectiveness planes. In addition, ICER acceptability curves will be presented and univariate sensitivity analyses will be performed focusing on uncertainty around most important costs-items.

\section{Dissemination policy}

To ensure optimal implementation we used the framework of Fleuren et al. [35]; consisting of patient, innovation, organization and socio-political determinants. Although clinical equipoise between surgery and ablation is reached for small CRLM, the results from recent meta-analyses, such as the most recent one by Meijerink et al. [36], do not support thermal ablation for resectable CRLM outside clinical trials. Hence, patients suitable for COLLISION will have to choose between surgery $(+/-$ ablation for unresectable CRLM) and trial participation.

With 15.549 new cases of colorectal cancer in the Netherlands (2015) approximately $4 \%$ of them will have $\geq 1$ resectable and ablatable CRLM [1]. At this moment, these lesions are treated by resection, whilst ablation may be associated with less complications and an equal or even superior oncological outcome. In other words, in the Netherlands alone an estimated target population of 625 patients per year should be eligible for COLLISION trial participation. To further facilitate implementation the trial is formally supported by the following concerning patient federations who joined the trial advisory board: The Dutch Federation for patients with cancer (NFK), the Dutch society for patients with gastro-intestinal and hepato-, pancreatico-, biliary cancers (SPKS) and the Dutch society for image-guided treatment of cancer (SBBvK).

The study is embedded within the multidisciplinary Dutch Colorectal Cancer Group (DCCG). DCCG is a collaboration between medical disciplines that are relevant for the diagnosis and treatment of colorectal cancer (surgical oncology, radiotherapy, medical oncology, pathology, radiology, gastroenterology, genetics). Patients will be recruited throughout the country and treated in one of the qualifying and selected high-volume centres. We will ensure that the scientific community, patients and professional organizations will be constantly kept up to date on the obtained results.

In order to qualify for reimbursement the Dutch health care institute (ZiNL) demands the best available evidence. Currently, thermal ablation is only approved for truly unresectable and small CRLM. Outside the setting of the trial ablations of resectable CRLM are off-guideline and hence not reimbursed. The direct and indirect costs of thermal ablation are considerably lower than that of surgery. We expect even lower indirect costs for patients treated within the study, primarily because thermal ablation of resectable CRLM in patients who by definition qualify as suitable for surgery may be associated with an even lower complication-rate.

\section{Discussion}

The recently published primary efficacy rates (complete ablation after the first procedure) of RFA and MWA for small CRLM have approached the reported resection 
plane recurrence rates for similar sized lesions [6]' [1417], [21]. Hence the issue of ablation site recurrences, that has previously prevented its widespread adoption, may be outdated. The relative ease to percutaneously re-ablate potential site recurrences, nowadays in the setting of a one-day admission under conscious sedation, has further downgraded its relevance.

\section{Partial hepatectomy}

Until relatively recent, patients with CRLM could only be cured by surgical resection of the lesions. Although no formal upper limit regarding number and size of CRLM has been established, surgical resection is nowadays considered safe and effective for patients with an adequate performance status if radical resection will leave sufficient future functioning liver parenchyma. In addition, one of the three main hepatic veins must be uncompromised and the liver remnant has to comprise a portal vein, hepatic artery and a bile duct. Clear definitions of what is regarded as resectable are lacking and vary dramatically from center to center on the basis of aggressiveness of the surgical team and the perception of the medical oncologist on when to refer patients [37]. To achieve consensus several societies for surgical oncology and hepatobiliary surgery have previously attempted to postulate resectability criteria (Table 2) [38, 39]. The objective of surgical resection for CRLM should be to remove all macroscopically visible tumour tissue with the intent to achieve cure. Histological tumour free margins and hence the confirmation of having radically resected the metastases remains essential.

Surgical resection has a 5-year OS reaching 31-58\% $[3,40]$. Although the number of serious adverse events of hepatic resection has decreased considerably in the past two decades, the 90-day mortality (4\%) and the complication-rate (40\%; major plus minor) are still high [41-43]. In 2007 data from 1059 non-cirrhotic patients who underwent major hepatectomy were analysed [43]. The total percentage of complications was 453 (43\%), divided as follows: minor complications 26\% (grade I 7\%; grade II 19\%) and major complications 17\% (grade IIIa $10 \%$, grade IIIb $2 \%$, grade IVa $4 \%$, grade IVb $1 \%$ ). Most frequently encountered complications include per-operative major bleeding, bile duct/gallbladder injury, perforation of adjacent structures, intra-abdominal infection, wound infection, liver abscess, haematoma at incision site, pneumothorax, liver failure and death (4\%) $[41,42]$.

\section{Radiofrequency ablation}

Since its introduction in the late 90's, RFA is the most studied and widely adopted ablative technique. It has emerged as a promising approach in the treatment of patients with unresectable CRLM. RFA has acquired its role in the treatment of patients with unresectable
CRLM as a safe, well tolerated, easily repeatable and less invasive procedure $[44,45]$.

One major drawback of RFA is the heat-sink effect in highly perfused organs, such as the liver where a large tumour located near large vessels $(>3 \mathrm{~mm}$ diameter) is not properly treated because heat is lost to the flowing blood. Another risk of RFA is heat injury to vital structures in or surrounding the ablated area. For this reason, treatment of lesions in the proximity of other organs, large vessels and major bile ducts has to be performed with caution, and is sometimes contra-indicated [46].

The 90-day mortality of thermal ablation alone is very low $(<1 \%)$ and the complication rate is also low $(6-9 \%)$ [6]. Applied to unresectable CRLM, 5-year survival rates are approaching the results reported after surgical resection, especially for patients presenting with a limited number of small-size lesions. The reported 5 -year OS is $25-55 \%[21-29]$. The recently presented long-term results from the only available randomized controlled trial shows a survival plateau of $36 \%$ after 8 -years in patients with unresectable CRLM [6]. It is important to realize that these percentages are derived from studies where thermal ablation was used to treat unresectable lesions. Ruers et al. found a PFS of 16.9 months (95\%CI 11.722.1) in a group of patients who received chemotherapy plus RFA (HR 0.63 [95\%CI 0.42-0.95]). Of those 56 patients treated with RFA, 9 developed a local site recurrence (LSR) (16,1\%) [6].

Complications can be divided into three different groups: related to probe placement (bleeding 0,7\%, infection, tumour seeding $0-0,3 \%$ ), related to energy delivery (damage to bowel, gallbladder, bile ducts $4,2 \%$, grounding pad burns, post-ablation syndrome, hepatic vascular damage, liver failure 2,1\%) and related to the general procedure (deep venous thrombosis, pulmonary embolism, referred pain, fever, nausea, vomiting, kidney failure) [47].

\section{Microwave ablation}

MWA is known as ablative technique for tissues with a high percentage of water and has several theoretical advantages that may result in improved performance near blood vessels. Due to a much broader field of power density, MWA results in a larger zone of active heating. This increased zone allows for a more homogeneous zone of tumour cell death, both within the targeted zone and next to blood vessels. This feature is thought to make MWA less affected by heat sink. Recent developments in the field of MWA, employing higher frequency bands $(2.45 \mathrm{GHz})$ or spatial energy control (thermal, field, and wavelength), claim to create more predictable, larger and more spherical ablation zones regardless of target location, tissue type or changes in tissue properties during the ablation [48]. 
Several studies reported a 3-,4- and 5-year OS for MWA between 35 and $79 \%, 35-58 \%$ and $17-18 \%$ $[15,16,49-54]$. M7ortality is ranging between 0 and $2 \%$ $[15,49,50]$. The median DFS ranges between 8 and 12 months $[15,50,54]$. Overall recurrence ranges between 39 and $72 \%[15,17,50,51,55,56]$. In several observational studies complications ranged between 0 and 54\% $[15,16,50,55-57]$. No studies reported the effect on quality of life after MWA.

\section{Partial hepatectomy versus thermal ablation}

Numerous studies reported OS rates for surgery and thermal ablation techniques. Comparing RFA alone to surgery alone numerous observational studies reported corrected hazard ratios for OS between RFA and surgery alone; treatment with RFA was associated with an inferior OS (HR = 1.92; 95\%CI 1.44-2.56) [22, 23, 25, 27, 5861]. Comparing RFA plus surgery to surgery alone other studies reported corrected hazard ratios and allowed for pooling between surgery and surgery plus RFA; no significant difference in OS was found $(\mathrm{HR}=1.29 ; 95 \% \mathrm{CI}$ 0.71-2.327) [14, 18, 61, 62].

For MWA, a 3-year OS of 23\% after surgery and 14\% after MWA has been reported [54]. Another study showed a 4-year OS of $70 \%$ after surgery and $41 \%$ after MWA, although no formal statistical comparison with surgery alone was reported [53]. A more recently published study found 5-year OS rates for surgery versus percutaneous ablation as first intervention of 51.9 and $53 \%$, with a median OS of 65.0 (95\%CI 47.3 to 82.6$)$ and 62.1 (95\%CI 52.2 to 72.1 ) months, respectively [19].

Another study reported no significant difference in OS for MWA plus surgery versus surgery alone (3-year OS: $50.9 \%$ vs $48.8 \%$ ) [63]. Median OS was 39 months after surgery and 28 months after MWA plus surgery. In multivariate analysis MWA was no prognostic factor for OS.

Several studies revealed that complications were significantly more common after surgery compared to RFA (relative risk $[R R]=0.47$; 95\%CI $0.28-0.78$ ) [22-29, 59, 64-66]. Two studies reported serious adverse events in $21-28 \%$ after surgery vs $13-37 \%$ in the surgery + ablation group $[18,24]$.

Some studies compared RFA to surgery alone regarding local progression-free survival (LPFS) and DFS; RFA was inferior to surgical resection (+/- RFA) [25, 28, 58]. Comparing RFA plus surgery to surgery alone, RFA plus surgery was associated with a poor LPFS [14, 18, 24, 58, 61]. Assessing DFS, no significant difference between RFA + surgery vs surgery alone was found.

In conclusion, a recently published systematic review and meta-analysis reported that further randomized assessments of thermal ablation with curative intent to current-day palliative chemotherapy alone should be considered unethical [36]. Therefore, the highest achievable evidence level for unresectable CRLM seems to be reached. According to above mentioned superior safety profile, lower complication-rate and competitive long term survival after thermal ablation for CRLM challenges liver surgery and fiats the setup of this randomized controlled trial. If thermal ablation for resectable CRLM proves to be non-inferior to surgery, a reduction of the post-procedural morbidity and mortality, length of hospital stay and incremental costs can be expected, with better quality of life and without compromising oncological outcome.

\begin{abstract}
Abbreviations
${ }^{18}$ F-FDG PET-CT: fludeoxyglucose F 18 positron emission tomographycomputed tomography; AE: Adverse events; ALT: Alanine transaminase; ASA: American Society of Anaesthesiologists; AST: Aspartate aminotransferase; ATE: Assisted technique efficacy; CEA: Carcinoembryonic antigen; Ce-CT: Contrast-enhanced computed tomography; Ce-MRI: Contrastenhanced Magnetic Resonance Imaging; CRB: Clinical research bureau; CRLM: Colorectal liver metastases; CRS: Clinical risk score; DCCG: Dutch Colorectal Cancer Group; DFS: Disease-free survival; DSMB: Data safety monitoring board; ECOG: Eastern Cooperative Oncology Group status; FLR: Future liver remnant; GCP: Good clinical practice; GHz: Gigahertz; HR: Hazard ratio; ICER: Incremental cost-effectiveness ratio; INR: International normalized ratio of prothrombin time of blood coagulation; IOUS: Intraoperative ultrasound; LPFS: Local progression-free survival; LSR: Local site recurrence; MWA: Microwave ablation; NDO: Number of drop-out; NI: Number of included patients; NLTFU: Number lost to follow-up; NR: Total number to be randomized; NS: Total sample size; NVIR: Dutch national society for interventional radiology; NVvR: Dutch national society for radiology; OS: Overall survival; $\mathrm{pE}$ : Probability of event; $\mathrm{PH}$ : Partial hepatectomy; PTE: Primary technique efficacy; QALY: Quality-adjusted life years; QoL: Quality of life; RFA: Radiofrequency ablation; RR: Relative risk; SADE: Serious adverse device effect; SAE: Serious adverse events; TTLP: Timeto-local-progression; TTP: Time-to-progression; ULN: Upper limit; VAS: Visual analogue scale; WHO: World Health Organization; WLC: liver surgery working
\end{abstract} group

\section{Acknowledgements}

The authors thank Karen Egging, Ires Joore, Janine Stolwijk, Carmilla Licht, Govert Mattijssen for their collaboration.

Authors.

Robbert S Puijk, Amsterdam UMC (location VUmc), Amsterdam, the Netherlands; Alette H Ruarus, Amsterdam UMC (location VUmc), Amsterdam, the Netherlands; Laurien GPH Vroomen, Amsterdam UMC (location VUmc), Amsterdam, the Netherlands; Aukje AJM van Tilborg, Amsterdam UMC (location VUmc), Amsterdam, the Netherlands; Hester J Scheffer, Amsterdam UMC (location VUmc), Amsterdam, the Netherlands; Karin Nielsen,

Amsterdam UMC (location VUmc), Amsterdam, the Netherlands; Marcus C de Jong, Amsterdam UMC (location VUmc), Amsterdam, the Netherlands; Jan JJ de Vries, Amsterdam UMC (location VUmc), Amsterdam, the Netherlands; Babs Zonderhuis, Amsterdam UMC (location VUmc), Amsterdam, the Netherlands; Hasan H. Eker, Amsterdam UMC (location VUmc), Amsterdam, the Netherlands; Geert Kazemier, Amsterdam UMC (location VUmc), Amsterdam, the Netherlands; Henk Verheul, Amsterdam UMC (location VUmc), Amsterdam, the Netherlands; Bram B van der Meijs, Amsterdam UMC (location VUmc), Amsterdam, the Netherlands; Laura van Dam, Amsterdam UMC (location VUmc), Amsterdam, the Netherlands; Natasha Sorgedrager, Amsterdam UMC (location VUmc), Amsterdam, the Netherlands; Veerle MH Coupé, Amsterdam UMC (location VUmc), Amsterdam, the Netherlands; Petrousjka MP van den Tol, Amsterdam UMC (location VUmc), Amsterdam, the Netherlands; Martijn R Meijerink, Amsterdam UMC (location VUmc), Amsterdam, the Netherlands.

COLLISION trial group

Co-authors who participated through the COLLISION collaborative group: Warner Prevoo, the Netherlands Cancer Institute, Antoni van Leeuwenhoek Ziekenhuis, Amsterdam, the Netherlands; Niels Kok, the Netherlands Cancer 
Institute, Antoni van Leeuwenhoek Ziekenhuis, Amsterdam, the Netherlands; Arjen L Diederik, Ziekenhuis Gelderse Vallei, Ede, the Netherlands; Gert Jan Spaargaren, Ziekenhuis Gelderse Vallei, Ede, the Netherlands; Colin Sietses, Ziekenhuis Gelderse Vallei, Ede, the Netherlands; Tjarda N van Heek, Ziekenhuis Gelderse Vallei, Ede, the Netherlands; GianPiero Serafino, Ziekenhuis Gelderse Vallei, Ede, the Netherlands; Jurgen J Futterer, RadboudUMC, Nijmegen, the Netherlands; Peter B van den Boezem, RadboudUMC, Nijmegen, the Netherlands; Martijn Stommel, RadboudUMC, Nijmegen, the Netherlands; Hans de Wilt, RadboudUMC, Nijmegen, the Netherlands; Mark Arntz, RadboudUMC, Nijmegen, the Netherlands; Sjoerd Jenniskens, RadboudUMC, Nijmegen, the Netherlands; Mark Besselink, Amsterdam UMC (location AMC), Amsterdam, the Netherlands; Otto M van Delden, Amsterdam UMC (location AMC), Amsterdam, the Netherlands; Thomas M van Gulik, Amsterdam UMC (location AMC), Amsterdam, the Netherlands; Pieter J Tanis, Amsterdam UMC (location AMC), Amsterdam, the Netherlands; Krijn P van Lienden, Amsterdam UMC (location AMC), Amsterdam, the Netherlands; Mark C Burgmans, Leids Universitair Medisch Centrum, Leiden, the Netherlands; Rutger-Jan Swijnenburg, Leids Universitair Medisch Centrum, Leiden, the Netherlands; Arian R van Erkel, Leids Universitair Medisch Centrum, Leiden, the Netherlands; Henk H Hartgrink, Leids Universitair Medisch Centrum, Leiden, the Netherlands; Jan Peringa, Onze Lieve Vrouwe Gasthuis, Amsterdam, the Netherlands; Hendrik Marsman, Onze Lieve Vrouwe Gasthuis, Amsterdam, the Netherlands; Peter CA Jacobs, Onze Lieve Vrouwe Gasthuis, Amsterdam, the Netherlands; Michael F Gerhards, Onze Lieve Vrouwe Gasthuis, Amsterdam, the Netherlands; Christiaan van der Leij, Maastricht Universitair Medisch Centrum, Maastricht, the Netherlands; Rutger Brans, Maastricht Universitair Medisch Centrum, Maastricht, the Netherlands; Marielle ME Coolsen, Maastricht Universitair Medisch Centrum, Maastricht, the Netherlands; Kees CHC Dejong, Maastricht Universitair Medisch Centrum, Maastricht, the Netherlands; Ronald van Dam, Maastricht Universitair Medisch Centrum, Maastricht, the Netherlands; Abbas Millad Solouki, Medisch Centrum Leewarden, Leeuwarden, the Netherlands; Johan A Dol, Medisch Centrum Leewarden, Leeuwarden, the Netherlands; Ted WF Vink, Medisch Centrum Leewarden, Leeuwarden, the Netherlands; Eric R Manusama, Medisch Centrum Leewarden, Leeuwarden, the Netherlands; Gijs A Patijn, Isala Klinieken, Zwolle, the Netherlands; Vincent B. Nieuwenhuijs, Isala Klinieken, Zwolle, the Netherlands; Mark A.J. Meijer, Isala Klinieken, Zwolle, the Netherlands; Hans Torrenga, Deventer Ziekenhuis, Deventer, the Netherlands; Eric D.J.A. Sonneveld, Westfriesgasthuis, Hoorn, the Netherlands; Jan-Willem W.D. de Waard, Westfriesgasthuis, Hoorn, the Netherlands; Joris J.A. Joosten "Westfriesgasthuis, Hoorn, the Netherlands; Cees Verhoef, Erasmus Medical Center, Rotterdam, the Netherlands; Adriaan Moelker, Erasmus Medical Center, Rotterdam, the Netherlands; Dirk Jan Grunhagen, Erasmus Medical Center, Rotterdam, the Netherlands; Bas Groot Koerkamp, Erasmus Medical Center, Rotterdam, the Netherlands; Jeroen Hagendoorn, Universitair Medisch Centrum, Utrecht, the Netherlands; I. Quintus Molenaar, Universitair Medisch Centrum, Utrecht, the Netherlands; Rutger CG Bruijnen, Universitair Medisch Centrum, Utrecht, the Netherlands; Karin CMJ van Nieuwkerk, Amsterdam UMC (location VUmc), Amsterdam, the Netherlands; Peter van de Ven, Amsterdam UMC (location VUmc), Amsterdam, the Netherlands; Astrid de Wind, EMGO+ Institute, Amsterdam, the Netherlands; Han Anema, EMGO + Institute, Amsterdam, the Netherlands; Jacob de Bakker, Amsterdam UMC (location VUmc), Amsterdam, the Netherlands; Martijn WH Leenders, Amsterdam UMC (location VUmc), Amsterdam, the Netherlands; Tessa Hellingman, Amsterdam UMC (location VUmc), Amsterdam, the Netherlands; Nicole van Grieken, Amsterdam UMC (location VUmc), Amsterdam, the Netherlands; Sanne Nieuwenhuizen, Amsterdam UMC (location VUmc), Amsterdam, the Netherlands; Bart Geboers, Amsterdam UMC (location VUmc), Amsterdam, the Netherlands; David J Breen, University Hospital of Southampton, Southampton, United Kingdom; Luca Aldrighetti, San Raffaele, Milan, Italy; Francesco De Cobelli, San Raffaele, Milan, Italy; Francesca Ratti, San Raffaele, Milan, Italy; Paolo Marra, San Raffaele, Milan, Italy; Thomas Albrecht, Vivantes Klinikum Neukölln, Berlin, Germany; PD Muller, Vivantes Klinikum Neukölln, Berlin, Germany; Cornelis van Kuijk, Amsterdam UMC (location VUmc), Amsterdam, the Netherlands.

\section{Funding}

Investigator Sponsored Research (ISR) grant by Medtronic PLC. The funders had no role in the design of the study; the collection, analysis, or interpretation of the data; the writing of the manuscript; or the decision to submit the manuscript for publication.

\section{Authors' contributions}

$\mathrm{RP}$ will be the study coordinator and was responsible for design of the trial, collecting data and writing of the manuscript. AR, LV, AT, HS, KN, MJ, JV, BZ, $H E, G K, H V, B M, L D, N S, V C$ and PVdT contributed to the conception and design of the study and reviewed the report. MM is the principal investigator of this trial and contributed to the trial conception and design, data collection and writing of the manuscript. All authors approved of the final version submitted.

\section{Ethics approval and consent to participate}

Ethics approval was obtained from the Medisch Ethische Toetsingscommissie (METc) Amsterdam UMC (location VUmc). Reference number 2016.561 NL-number NL58551.029.16. All participants will provide written informed consent.

\section{Consent for publication}

Not applicable.

\section{Competing interests}

Investigator Sponsored Research (ISR) grant by Medtronic PLC. The funders had no role in the design of the study; the collection, analysis, or interpretation of the data; the writing of the manuscript; or the decision to submit the manuscript for publication.

\section{Publisher's Note}

Springer Nature remains neutral with regard to jurisdictional claims in published maps and institutional affiliations.

\section{Author details}

'Department of Radiology and Nuclear Medicine, Amsterdam University Medical Centres (location VUmc), de Boelelaan 1117, 1081, HV, Amsterdam, The Netherlands. ${ }^{2}$ Department of Surgery, Amsterdam University Medical Centres (location VUmc), de Boelelaan 1117, 1081, HV, Amsterdam, The Netherlands. ${ }^{3}$ Department of Medical Oncology, Amsterdam University Medical Centres (location VUmc), de Boelelaan 1117, 1081, HV, Amsterdam, The Netherlands. ${ }^{4}$ Department of Epidemiology and Biostatistics, Amsterdam University Medical Centres (location VUmc), de Boelelaan 1117, 1081, HV, Amsterdam, The Netherlands.

Received: 30 June 2017 Accepted: 2 August 2018

Published online: 15 August 2018

\section{References}

1. Comprehensive Cancer Organisation the Netherlands (I.K.N.L.). National evidence-based guideline. Colorectaalcarcinoom. Available from: https://www.oncoline.nl/colorectaalcarcinoom.

2. Ferlay J, Shin HR, Bray F, Forman D, Mathers C, Parkin DM. Estimates of worldwide burden of cancer in 2008: GLOBOCAN 2008. Int J Cancer. 2010; 127(12):2893-917.

3. Belinson S, Chopra R, Yang Y, Shankaran V, Aronson N. Local Hepatic Therapies for Metastases to the Liver From Unresectable Colorectal Cancer. AHRQ Comparative Effectiveness Reviews. Rockville: Agency for Healthcare Research and Quality (US); 2012. Report No.: 13-EHC014-EF.

4. Smith MD, McCall JL. Systematic review of tumour number and outcome after radical treatment of colorectal liver metastases. Br J Surg. 2009;96(10): 1101-13.

5. Sutherland LM, Williams JA, Padbury RT, Gotley DC, Stokes B, Maddern GJ. Radiofrequency ablation of liver tumors: a systematic review. Arch Surg. 2006;141(2):181-90.

6. Ruers T, Van Coevorden F, Punt CJ, Pierie JE, Borel-Rinkes I, Ledermann JA, Poston G, Bechstein W, Lentz MA, Mauer M, et al. Local Treatment of Unresectable Colorectal Liver Metastases: Results of a Randomized Phase II Trial. J Natl Cancer Inst. 2017;109(9):1-10.

7. Puijk RS, Ruarus AH, Scheffer HJ, Vroomen L, van Tilborg A, de Vries JJJ, Berger FH, van den Tol PMP, Meijerink MR. Percutaneous liver tumour ablation: image guidance, endpoint assessment, and quality control. Can Assoc Radiol J. 2018;69(1):51-62.

8. Van Tilborg AA, Meijerink MR, Sietses C, Van Waesberghe JH, Mackintosh MO, Meijer S, Van Kuijk C, Van Den Tol P. Long-term results of radiofrequency ablation for unresectable colorectal liver metastases: a potentially curative intervention. Br J Radiol. 2011;84(1002):556-65. 
9. Bengmark S, Hafstrom L. The natural history of primary and secondary malignant tumors of the liver. I. The prognosis for patients with hepatic metastases from colonic and rectal carcinoma by laparotomy. Cancer. 1969; 23(1):198-202

10. Goldberg RM. Therapy for metastatic colorectal cancer. Oncologist. 2006; 11(9):981-7.

11. Hurwitz H, Fehrenbacher L, Novotny W, Cartwright T, Hainsworth J, Heim W, Berlin J, Baron A, Griffing S, Holmgren E, et al. Bevacizumab plus irinotecan, fluorouracil, and leucovorin for metastatic colorectal cancer. N Engl J Med. 2004;350(23):2335-42.

12. Mulier S, Ruers T, Jamart J, Michel L, Marchal G, Ni Y. Radiofrequency ablation versus resection for resectable colorectal liver metastases: time for a randomized trial? An update. Dig Surg. 2008;25(6):445-60.

13. Forner A, Llovet JM, Bruix J. Hepatocellular carcinoma. Lancet. 2012; 379(9822):1245-55

14. Eltawil KM, Boame N, Mimeault R, Shabana W, Balaa FK, Jonker DJ, Asmis TR, Martel G. Patterns of recurrence following selective intraoperative radiofrequency ablation as an adjunct to hepatic resection for colorectal liver metastases. J Surg Oncol. 2014;110(6):734-8.

15. Stattner S, Jones RP, Yip VS, Buchanan K, Poston GJ, Malik HZ, Fenwick SW. Microwave ablation with or without resection for colorectal liver metastases. Eur J Surg Oncol. 2013;39(8):844-9.

16. Groeschl RT, Wong RK, Quebbeman EJ, Tsai S, Turaga KK, Pappas SG, Christians KK, Hohenwalter EJ, Tutton SM, Rilling WS, et al. Recurrence after microwave ablation of liver malignancies: a single institution experience. HPB : the official journal of the International Hepato Pancreato Biliary Association. 2013;15(5):365-71.

17. Groeschl RT, Pilgrim CH, Hanna EM, Simo KA, Swan RZ, Sindram D, Martinie JB, lannitti DA, Bloomston M, Schmidt C, et al. Microwave ablation for hepatic malignancies: a multiinstitutional analysis. Ann Surg. 2014;259(6): 1195-200.

18. Faitot F, Faron M, Adam R, Elias D, Cimino M, Cherqui D, Vibert E, Castaing D, Cunha AS, Goere D. Two-stage hepatectomy versus 1-stage resection combined with radiofrequency for bilobar colorectal metastases: a casematched analysis of surgical and oncological outcomes. Ann Surg. 2014 260(5):822-7. discussion 827-828

19. Hof J, Wertenbroek MW, Peeters PM, Widder J, Sieders E, de Jong KP. Outcomes after resection and/or radiofrequency ablation for recurrence after treatment of colorectal liver metastases. Br J Surg. 2016;103(8):1055-62.

20. Karanicolas PJ, Jarnagin WR, Gonen M, Tuorto S, Allen PJ, DeMatteo RP, D'Angelica MI, Fong Y. Long-term outcomes following tumor ablation for treatment of bilateral colorectal liver metastases. JAMA Surg. 2013;148(7): 597-601.

21. Nielsen $K$, van Tilborg AA, Meijerink MR, Macintosh MO, Zonderhuis BM, de Lange ES, Comans EF, Meijer S, van den Tol MP. Incidence and treatment of local site recurrences following RFA of colorectal liver metastases. World J Surg. 2013;37(6):1340-7.

22. Agcaoglu O, Aliyev S, Karabulut K, El-Gazzaz G, Aucejo F, Pelley R, Siperstein $\mathrm{AE}$, Berber $\mathrm{E}$. Complementary use of resection and radiofrequency ablation for the treatment of colorectal liver metastases: an analysis of 395 patients. World J Surg. 2013;37(6):1333-9.

23. Aliyev S, Agcaoglu O, Aksoy E, Taskin HE, Vogt D, Fung J, Siperstein A, Berber E. Efficacy of laparoscopic radiofrequency ablation for the treatment of patients with small solitary colorectal liver metastasis. Surgery. 2013; 154(3):556-62.

24. Kim KH, Yoon YS, Yu CS, Kim TW, Kim HJ, Kim PN, Ha HK, Kim JC. Comparative analysis of radiofrequency ablation and surgical resection for colorectal liver metastases. J Korean Surg Soc. 2011;81(1):25-34.

25. Hur H, Ko YT, Min BS, Kim KS, Choi JS, Sohn SK, Cho CH, Ko HK, Lee JT, Kim NK. Comparative study of resection and radiofrequency ablation in the treatment of solitary colorectal liver metastases. Am J Surg. 2009;197(6):728-36.

26. McKay A, Fradette K, Lipschitz J. Long-term outcomes following hepatic resection and radiofrequency ablation of colorectal liver metastases. HPB Surg. 2009;2009:346863.

27. Berber $\mathrm{E}$, Tsinberg M, Tellioglu G, Simpfendorfer $\mathrm{CH}$, Siperstein AE. Resection versus laparoscopic radiofrequency thermal ablation of solitary colorectal liver metastasis. J Gastrointest Surg. 2008;12(11):1967-72.

28. Nishiwada S, Ko S, Mukogawa T, Ishikawa H, Matsusaka M, Nakatani T, Kikuchi E, Watanabe A. Comparison between percutaneous radiofrequency ablation and surgical hepatectomy focusing on local disease control rate for colorectal liver metastases. Hepatogastroenterology. 2014;61(130):436-41.
29. Otto G, Duber C, Hoppe-Lotichius M, Konig J, Heise M, Pitton MB. Radiofrequency ablation as first-line treatment in patients with early colorectal liver metastases amenable to surgery. Ann Surg. 2010;251(5):796-803.

30. Curley SA. Radiofrequency ablation versus resection for resectable colorectal liver metastases: time for a randomized trial? Ann Surg Oncol. 2008;15(1):11-3.

31. Mulier S, Ni Y, Jamart J, Michel L, Marchal G, Ruers T. Radiofrequency ablation versus resection for resectable colorectal liver metastases: time for a randomized trial? Ann Surg Oncol. 2008;15(1):144-57.

32. Sietses C, Meijerink MR, Meijer S, van den Tol MP. The impact of intraoperative ultrasonography on the surgical treatment of patients with colorectal liver metastases. Surg Endosc. 2010;24(8):1917-22.

33. Castor EDC. Amsterdam, the Netherlands. 2018. https://www.castoredc.com/ Accessed 10 Apr 2018.

34. Crocetti L, de Baere T, Lencioni R. Quality improvement guidelines for radiofrequency ablation of liver tumours. Cardiovasc Intervent Radiol. 2010; 33(1):11-7.

35. Fleuren $M$, Wiefferink $K$, Paulussen $T$. Determinants of innovation within health care organizations: literature review and Delphi study. Int J Qual Health Care. 2004;16(2):107-23.

36. Meijerink MR, Puijk RS, van Tilborg A, Henningsen KH, Fernandez LG, Neyt M, Heymans J, Frankema JS, de Jong KP, Richel DJ, et al. Radiofrequency and microwave ablation compared to systemic chemotherapy and to partial hepatectomy in the treatment of colorectal liver metastases: a systematic review and meta-analysis. Cardiovasc Intervent Radiol. 2018;41(8):1189-204.

37. Choti MA, Thomas M, Wong SL, Eaddy M, Pawlik TM, Hirose K, Weiss MJ, Kish J, Green MR. Surgical resection preferences and perceptions among medical oncologists treating liver metastases from colorectal Cancer. Ann Surg Oncol. 2016;23(2):375-81.

38. Rees M, Elias D, Coimbra FJ, Orloff SL, Americas Hepato-Pancreato-Biliary A Society of Surgical O, Society for Surgery of the alimentary T. Selection for hepatic resection: expert consensus conference. HPB : the official journal of the International Hepato Pancreato Biliary Association. 2013:15(2):104-5.

39. Jones RP, Vauthey JN, Adam R, Rees M, Berry D, Jackson R, Grimes N, Fenwick SW, Poston GJ, Malik HZ. Effect of specialist decision-making on treatment strategies for colorectal liver metastases. Br J Surg. 2012;99(9): 1263-9.

40. Cirocchi R, Trastulli S, Boselli C, Montedori A, Cavaliere D, Parisi A, Noya G, Abraha I. Radiofrequency ablation in the treatment of liver metastases from colorectal cancer. Cochrane Database Syst Rev. 2012;6:CD006317.

41. van der Pool AE, Damhuis RA, ljzermans JN, de Wilt JH, Eggermont AM, Kranse $R$, Verhoef $C$. Trends in incidence, treatment and survival of patients with stage IV colorectal cancer: a population-based series. Color Dis. 2012; 14(1):56-61.

42. Dols LF, Verhoef C, Eskens FA, Schouten O, Nonner J, Hop WC, Mendez Romero A, de Man RA, van der Linden E, Dwarkasing RS, et al. Improvement of 5 year survival rate after liver resection for colorectal metastases between 1984-2006. Ned Tijdschr Geneeskd. 2009:153(11):490-5.

43. Mullen JT, Ribero D, Reddy SK, Donadon M, Zorzi D, Gautam S, Abdalla EK, Curley SA, Capussotti L, Clary BM, et al. Hepatic insufficiency and mortality in 1,059 noncirrhotic patients undergoing major hepatectomy. J Am Coll Surg. 2007;204(5):854-62. discussion 862-854

44. Solbiati L, lerace T, Goldberg SN, Sironi S, Livraghi T, Fiocca R, Servadio G, Rizzatto G, Mueller PR, Del Maschio A, et al. Percutaneous US-guided radiofrequency tissue ablation of liver metastases: treatment and follow-up in 16 patients. Radiology. 1997:202(1):195-203.

45. Berber E, Siperstein AE. Perioperative outcome after laparoscopic radiofrequency ablation of liver tumors: an analysis of 521 cases. Surg Endosc. 2007;21(4):613-8

46. Wood TF, Rose DM, Chung M, Allegra DP, Foshag LJ, Bilchik AJ. Radiofrequency ablation of 231 unresectable hepatic tumors: indications, limitations, and complications. Ann Surg Oncol. 2000;7(8):593-600.

47. Rhim H, Lim HK, Kim YS, Choi D, Lee WJ. Radiofrequency ablation of hepatic tumors: lessons learned from 3000 procedures. J Gastroenterol Hepatol. 2008:23(10):1492-500

48. Lencioni R, de Baere T, Martin RC, Nutting CW, Narayanan G. Image-guided ablation of malignant liver tumors: recommendations for clinical validation of novel thermal and non-thermal technologies - a western perspective. Liver Cancer. 2015:4(4):208-14.

49. Leung U, Kuk D, D'Angelica MI, Kingham TP, Allen PJ, DeMatteo RP, Jarnagin WR, Fong Y. Long-term outcomes following microwave ablation for liver malignancies. Br J Surg. 2015;102(1):85-91. 
50. Martin RC, Scoggins CR, McMasters KM. Safety and efficacy of microwave ablation of hepatic tumors: a prospective review of a 5-year experience. Ann Surg Oncol. 2010;17(1):171-8.

51. Seki T, Wakabayashi M, Nakagawa T, Imamura M, Tamai T, Nishimura A, Yamashiki N, Inoue K. Percutaneous microwave coagulation therapy for solitary metastatic liver tumors from colorectal cancer: a pilot clinical study. Am J Gastroenterol. 1999;94(2):322-7.

52. Wang J, Liang P, Yu J, Yu MA, Liu F, Cheng Z, Yu X. Clinical outcome of ultrasound-guided percutaneous microwave ablation on colorectal liver metastases. Oncol Lett. 2014;8(1):323-6.

53. Engstrand J, Nilsson $\mathrm{H}$, Jansson A, Isaksson B, Freedman J, Lundell L, Jonas E. A multiple microwave ablation strategy in patients with initially unresectable colorectal cancer liver metastases - a safety and feasibility study of a new concept. Eur J Surg Oncol. 2014;40(11):1488-93.

54. Shibata T, Niinobu T, Ogata N, Takami M. Microwave coagulation therapy for multiple hepatic metastases from colorectal carcinoma. Cancer. 2000; 89(2):276-84.

55. Eng OS, Tsang AT, Moore D, Chen C, Narayanan S, Gannon CJ, August DA Carpizo DR, Melstrom LG. Outcomes of microwave ablation for colorectal cancer liver metastases: a single center experience. J Surg Oncol. 2015; 111(4):410-3.

56. Correa-Gallego C, Fong Y, Gonen M, D'Angelica MI, Allen PJ, DeMatteo RP, Jarnagin WR, Kingham TP. A retrospective comparison of microwave ablation vs. radiofrequency ablation for colorectal cancer hepatic metastases. Ann Surg Oncol. 2014;21(13):4278-83.

57. Liang PC, Lai HS, Shih TT, Wu CH, Huang KW. The pilot experience upon surgical ablation of large liver tumor by microwave system with tissue permittivity feedback control mechanism. BMC Surg. 2014;14:82.

58. Abdalla EK, Vauthey JN, Ellis LM, Ellis V, Pollock R, Broglio KR, Hess K, Curley $S A$. Recurrence and outcomes following hepatic resection, radiofrequency ablation, and combined resection/ablation for colorectal liver metastases. Ann Surg. 2004;239(6):818-25. discussion 825-817

59. Jasarovic D, Stojanovic D, Mitrovic N, Stevanovic D. Resection or radiofrequency ablation of colorectal liver metastasis. Vojnosanit Pregl. 2014; 71(6):542-6.

60. Lee KH, Kim HO, Yoo CH, Son BH, Park YL, Cho YK, Kim H, Han WK. Comparison of radiofrequency ablation and resection for hepatic metastasis from colorectal cancer. Korean J Gastroenterol. 2012;59(3):218-23.

61. Gleisner AL, Choti MA, Assumpcao L, Nathan H, Schulick RD, Pawlik TM. Colorectal liver metastases: recurrence and survival following hepatic resection, radiofrequency ablation, and combined resection-radiofrequency ablation. Arch Surg. 2008;143(12):1204-12.

62. Boame N, Gresham G, Jonker D, Martel G, Balaa F, Asmis T. Use of chemotherapy and radiofrequency ablation to treat colorectal cancer metastases: a retrospective review of the Ottawa Hospital Cancer Centre over 7 years. Curr Oncol. 2014;21(4):e557-63.

63. Tanaka K, Shimada H, Nagano Y, Endo I, Sekido H, Togo S. Outcome after hepatic resection versus combined resection and microwave ablation for multiple bilobar colorectal metastases to the liver. Surgery. 2006;139(2):263-73.

64. Aloia TA, Vauthey JN, Loyer EM, Ribero D, Pawlik TM, Wei SH, Curley SA, Zorzi D, Abdalla EK. Solitary colorectal liver metastasis: resection determines outcome. Arch Surg. 2006;141(5):460-6. discussion 466-467

65. Lee H, Heo JS, Cho YB, Yun SH, Kim HC, Lee WY, Choi SH, Choi DW. Hepatectomy vs radiofrequency ablation for colorectal liver metastasis: a propensity score analysis. World J Gastroenterol. 2015;21(11):3300-7.

66. Lee WS, Yun SH, Chun HK, Lee WY, Kim SJ, Choi SH, Heo JS, Joh JW, Choi D, Kim SH, et al. Clinical outcomes of hepatic resection and radiofrequency ablation in patients with solitary colorectal liver metastasis. J Clin Gastroenterol. 2008;42(8):945-9.

Ready to submit your research? Choose BMC and benefit from:

- fast, convenient online submission

- thorough peer review by experienced researchers in your field

- rapid publication on acceptance

- support for research data, including large and complex data types

- gold Open Access which fosters wider collaboration and increased citations

- maximum visibility for your research: over $100 \mathrm{M}$ website views per year

At BMC, research is always in progress.

Learn more biomedcentral.com/submissions 